\title{
Associação do Valor Preditivo do New Injury Severity Score (NISS) Com A Escala de Resultados de Glasgow em Pacientes Com Lesão Axonial Difusa
}

\author{
Cristiane de Alencar Domingues, Rita de Cássia Almeida Vieira, Almir Ferreira de Andrade, \\ Vinícius Monteiro de Paula Guirado, Regina Márcia Cardoso de Sousa
}

\begin{abstract}
Traumatic brain injury (TBI) affects large numbers of victims and constitutes a serious public health problem in Brazil. Diffuse axonal injury (DAI) is the most common consequence of TBI and it is responsible for most of the chronic sequelae. Analysis of the prognostic index new injury severity score (NISS) determines the severity of injuries by body region examined during the hospital stay and it is really useful in making decisions about treatment strategies and to predict mortality of victims. Given the above, the purpose of this study was to collect epidemiological data of victims with exclusive diagnosis of DAl and test the association of the predictive value of the NISS with the Glasgow Outcome Scale (GOS) at the hospital discharge. We analyzed medical records of 21 victims with exclusive diagnosis of DAl admitted to the DAl ambulatory at Clinics Hospital of the Faculty of Medicine, University of São Paulo, located in São Paulo city, from January 2011 to February 2012. The results show a predominance of male $(76.2 \%)$ aged 24 years $( \pm 11.9)$ admitted during the morning $(42.8 \%)$, on Saturday $(23.8 \%)$, victims of motorcycle accidents $(7442 \%)$, referred to the hospital by prehospital specialized service $(85.7 \%)$, with Glasgow Coma Scale less than or equal to $8(76.2 \%) ; 71.4 \%$ of the victims were addmited at the Intensive Care Unit (ICU), with an lenght of hospital stay of 07 days $( \pm 20.46)$ and NISS mean of $36( \pm 9.99)$. All victims had serious head injury with elevated head MAIS and severe disability $(66.7 \%)$. Statistically significant association was found between NISS and the GOS. The severity of the victims with DAI by NISS is an important indicator of health care and can assist in the planning of multidisciplinary rehabilitation program specialist.
\end{abstract}

Keywords: Brain injuries, Diffuse axonal injury, Trauma indexes, Wounds and injuries.

How to cite this article: de Alencar Domingues $C$, de Cássia Almeida Vieira R, de Andrade AF, de Paula Guirado VM, de Sousa RMC. Associação do Valor Preditivo do New Injury Severity Score (NISS) Com A Escala de Resultados de Glasgow em Pacientes Com Lesão Axonial Difusa. Panam J Trauma Critical Care Emerg Surg 2012;1(3):186-187.

Source of support: Nil

Conflict of interest: None declared

\section{RESUMEN}

O traumatismo craniencefálico (TCE) atinge grande número de vítimas e se constitui um grave problema de saúde pública no Brasil e no mundo. A Lesão Axonial Difusa (LAD) é a consequência mais comum das vítimas de TCE e é responsável pela maior parte das sequelas crônicas. As lesões traumáticas craniencefálicas causam alterações cognitivas, físicas e comportamentais, oneram o sistema de saúde e podem comprometer a qualidade de vida das vítimas e familiares.
Diante do exposto, o objetivo do estudo foi levantar dados epidemiológicos de vítimas com diagnóstico exclusivo de LAD e verificar a associação do valor preditivo do new injury severity score (NISS) com a Escala de Resultados de Glasgow (ERGI) no momento da alta hospitalar. Trata-se de um estudo descritivo, transversal com abordagem quantitativa, realizado no ambulatório de LAD do Hospital das Clínicas da Faculdade de Medicina da Universidade de São Paulo, localizado no município de São Paulo. A amostragem foi não probabilística por acessibilidade e constituiu-se de 21 vítimas de TCE com diagnóstico exclusivo de LAD, que atenderam aos seguintes critérios de inclusão: possuírem diagnóstico exclusivo de LAD e serem atendidas no ambulatório de LAD após a alta hospitalar; idade $\geq 18$ e $<60$ anos. Houve exclusão das vítimas que apresentavam distúrbios de comunicação verbal no período de coleta de dados. Os dados foram coletados entre janeiro de 2011 e fevereiro de 2012. Prontuários de todos os indivíduos cadastrados no ambulatório em 2011 e fevereiro de 2012 foram analisados tendo-se em vista os critérios de inclusão do estudo. Dessa análise restaram 21 vítimas. No prontuário foram coletados dados relacionados à: idade, gênero, internação, tipo e mecanismo do trauma, atendimento pré-hospitalar, internação em Unidade de Terapia Intensiva (UTI), tempo de permanência hospitalar, Escala de Coma e de Resultado de Glasgow, gravidade do trauma e do TCE. A gravidade do trauma foi estimada pelo new injury severity score (NISS) e a Maximum Abbreviated Injury Scale da região cabeça (MAIS/ Cabeça) foi aplicada para caracterizar a gravidade do TCE. As Escalas de Coma e de Resultado de Glasgow têm sido recomendadas para avaliar a capacidade funcional global em vítimas de TCE. O instrumento é de fácil aplicação, composto por cinco categorias para classificação dos pacientes: boa recuperação; incapacidade moderada; incapacidade grave; estado vegetativo persistente e morte. As informações relacionadas a esta investigação foram armazenados em banco de dados computadorizado do Programa Statistical Package for the Social Sciences (SPSS) versão 17,0. As variáveis numéricas foram primeiramente analisadas segundo o tipo de distribuição pelo teste de Shapiro Wilk. $\mathrm{Na}$ análise da associação dos resultados entre ERGI e a variável NISS utilizou-se o teste de Correlação de Pearson. Em todos os testes estatísticos foi adotado o nível de significância de 5\%. Os resultados evidenciaram predominância de homens (76,2\%) com idade média de 24 anos $( \pm 11,9)$, admitidos no período diurno manhã $(42,8 \%)$, no sábado $(23,8 \%)$, vítimas de acidente motociclístico $(74,42 \%)$, encaminhadas ao hospital por unidade pré-hospitalar especializada (85,7\%), com Escala de Coma de Glasgow menor ou igual a $8(76,2 \%)$. Um total de 15 vítimas $(71,4 \%)$ foram admitidas em UTI, a média de permanência hospitalar foi de 07 dias (dp = 20,46) e, do NISS, 36 (dp = 9,99 ). Todas as vítimas apresentaram escore MAIS/cabeça igual ou superior a 4 , indicativo de lesão grave. Destes $66,7 \%$ apresentaram incapacidade grave de acordo com a ERGI. Houve associação significativamente estatística entre o NISS

Paper presented at the student competition, 25th Annual Congress of Panamerican Trauma Society, Medellin, Colombia, November 2012. 
e a escala de resultado de Glasgow $(p=0,04)$. Os resultados obtidos nesta investigação indicam que o escore da gravidade das vítimas de LAD pelo NISS é um importante indicador dos cuidados à saúde e auxilia no planejamento de programa de reabilitação multidisciplinar especializado. A partir destes achados, a equipe multiprofissional pode planejar ações que visem contribuir para a reabilitação das vítimas de TCE e assim melhorar a qualidade de vida destas pessoas.

Palavras chaves: Traumatismos encefálicos, Lesão axonial difusa, Índices de trauma, Ferimentos e lesões.

\section{ACERCA DE LOS AUTORES}

\section{Cristiane de Alencar Domingues}

(Correspondiente Autor)

Enfermeira. Doutoranda do Programa de Pós-Graduação em Enfermagem na Saúde do Adulto da Escola de Enfermagem da Universidade de São Paulo, Brazil, e-mail: crismingues@usp.br

\section{Rita de Cássia Almeida Vieira}

Enfermeira. Doutoranda do Programa de Pós-Graduação em Enfermagem na Saúde do Adulto da Escola de Enfermagem da Universidade de São Paulo, Brazil

\section{Almir Ferreira de Andrade}

Médico Professor Livre-Docente do Departamento de Neurologia da Faculdade de Medicina da Universidade de São Paulo, Brazil

\section{Vinícius Monteiro de Paula Guirado}

Médico. Neurocirurgião do Hospital das Clínicas da Faculdade de Medicina da Universidade de São Paulo, Brazil

\section{Regina Márcia Cardoso de Sousa}

Enfermeira. Professora Titular do Departamento de Enfermagem Médico-Cirúrgica da Escola de Enfermagem da Universidade de São Paulo, Brazil 